\title{
Synthesis of Acyclic and Cyclic Sulfamates: A Review
}

\author{
Hamid Saeidian* \\ Morteza Abdoli \\ Zohreh Mirjafary \\ Synthesis 2015, 47, 1057.
}

Please note that for the final print and online versions a 'Note Added in Proof' was inserted, including Table 7 and the corresponding references. 